\title{
EUCLIDEAN, HYPERBOLIC AND SPHERICAL BLOCH CONSTANTS
}

\author{
BY C. DAVID MINDA ${ }^{1}$ \\ TO PROFESSOR S. E. WARSCHAWSKI ON HIS 78TH BIRTHDAY
}

We begin with a brief survey of some of the known results dealing with Bloch constants. Bloch's theorem [3] asserts that there is a constant $B_{1}>0$ such that if $f$ is holomorphic in the open unit disk B and normalized by $\left|f^{\prime}(0)\right| \geqslant 1$, then the Riemann surface of $f$, viewed as spread over the complex plane $\mathbf{C}$, contains an unramified disk of radius at least $B_{1}$. Pommerenke [11] introduced the locally schlicht Bloch constant $B_{\infty}>B_{1}$ which has the same property relative to the family of normalized locally schlicht holomorphic functions defined on $\mathbf{B}$. He showed that $B_{\infty} \leqslant \mathfrak{R}$, where $\mathfrak{R}$ denotes the Landau constant [7]. The precise values of these constants are not known; however, the following bounds are known.

$$
\begin{gathered}
.433<\frac{\sqrt{3}}{4}<B_{1} \leqslant \frac{1}{\sqrt{1+\sqrt{3}}} \frac{\Gamma(1 / 3) \Gamma(11 / 12)}{\Gamma(1 / 4)}<.4719, \\
\frac{1}{2}<B_{\infty} \leqslant \& \leqslant \frac{\Gamma(1 / 3) \Gamma(5 / 6)}{\Gamma(1 / 6)}<.5433 .
\end{gathered}
$$

The lower bounds for $B_{1}$ and $B_{\infty}$, but without strict inequality, are the work of Ahlfors [1]. The strict inequalities were established by Heins [6] and Pommerenke [11], respectively. The upper bound for the Bloch constant comes from an example of Ahlfors and Grunsky [2] and is conjectured to be sharp. The upper bound for the Landau constant that is frequently cited is .544; this is due to an unpublished example of R. M. Robinson that is mentioned in [1]. In [8] we present an explicit example which yields the upper bound for the Landau constant that is given above. It is analogous to the Ahlfors-Grunsky example and it is plausible that it should give the actual value of the Landau constant. It is probably the example of Robinson. In fact, in [8] we exhibit a unified approach to obtaining upper and lower bounds for these and other new Bloch constants. As special cases of our results we obtain all of the previously mentioned bounds.

Received by the editors September 11, 1981.

1980 Mathematics Subject Classification. Primary 30C25, 30C80.

${ }^{1}$ This research was completed while the author was visiting the University of California, San Diego during 1980-81.

(C) 1982 American Mathematical Society 0273-0979/81/0000-0085/\$02.00 
Before we can define the various Bloch constants, we must establish certain notation and terminology. Let $\mathbf{P}$ denote the Riemann sphere. Suppose $f: \mathbf{B} \longrightarrow$ $\Omega$ is a meromorphic function, where $\Omega$ is one of $\mathbf{B}, \mathbf{C}$ and $\mathbf{P}$. Of course, if $\Omega$ is B or $\mathrm{C}$, then $f$ is actually holomorphic. We view $R_{f}$, the Riemann surface of $f$, as being spread over $\Omega$. In order to measure the size of unramified disks on $R_{f}$, we need to fix a distance function on $\Omega$. Set

$$
\begin{aligned}
& \delta_{\mathbf{B}}(z, w)=\left|\frac{z-w}{1-\bar{w} z}\right| \quad \text { if } z, w \in \mathbf{B}, \\
& \delta_{\mathbf{C}}(z, w)=|z-w| \quad \text { if } z, w \in \mathbf{C}, \\
& \delta_{\mathbf{P}}(z, w)=\left|\frac{z-w}{1+\bar{w} z}\right| \quad \text { if } z, w \in \mathbf{P} .
\end{aligned}
$$

Then $\delta_{\mathbf{B}}$ and $\delta_{\mathrm{C}}$ are true distance functions while $\delta_{\mathrm{P}}$ is not. However, $2 \tan ^{-1}\left(\delta_{\mathrm{P}}(z, w)\right)$ does give the spherical distance between $z$ and $w$, so $\delta_{\mathbf{P}}$ is closely related to a distance function. In any case, we shall use $\delta_{\Omega}(\Omega=\mathbf{B}$, $\mathbf{C}$ or $\mathbf{P}$ ) to measure the radius of disks on $\Omega$. The reason for doing this is that the close analogy between bounds for the various Bloch constants will be apparent. For $z \in \mathbf{B}$ let $r_{\Omega}(z, f)$ denote the radius, relative to $\delta_{\Omega}$, of the largest unramified disk in $R_{f}$ having center $f(z)$. Define $r_{\Omega}(f)=\sup \left\{r_{\Omega}(z, f): z \in \mathbf{B}\right\}$. Set

$$
f^{\Omega}(0)= \begin{cases}\frac{\left|f^{\prime}(0)\right|}{1-|f(0)|^{2}} & \text { if } \Omega=\mathbf{B}, \\ \left|f^{\prime}(0)\right| & \text { if } \Omega=\mathbf{C}, \\ \frac{\left|f^{\prime}(0)\right|}{1+|f(0)|^{2}} & \text { if } \Omega=\mathbf{P} .\end{cases}
$$

For $m \in \mathbf{Z}^{+} \cup\{\infty\}$ let $F_{m}(\mathbf{B}, \Omega)$ denote the family of all nonconstant meromorphic functions $f: \mathbf{B} \rightarrow \Omega$ such that for each $q \in f(\mathbf{B})$ each root of $f=q$ is either simple or else has multiplicity at least $m+1$. For $m=1$ this is just the family of all nonconstant meromorphic mappings of $\mathbf{B}$ into $\Omega$, while for $m=\infty$ this gives locally schlicht meromorphic functions.

Now we can introduce the various Bloch constants. For $\alpha>0$ let

$$
B_{m, \Omega}(\alpha)=\inf \left\{r_{\Omega}(f): f \in F_{m}(\mathbf{B}, \Omega) \text { and } f^{\Omega}(0) \geqslant \alpha\right\} \text {. }
$$

It is straightforward to check that $B_{m, C}(\alpha)=\alpha B_{m, C}(1)$. Notice that $B_{1, C}(1)$ is the classical Bloch constant and that $B_{\infty, C}(1)$ is the locally schlicht Bloch constant. In the hyperbolic case $(\Omega=\mathrm{B})$ or the spherical case $(\Omega=\mathrm{P})$, the Bloch constants are not linear functions of $\alpha$. 
We obtain lower bounds for these hyperbolic, euclidean and spherical Bloch constants. These bounds are established by making use of a differentialgeometric method that was introduced by Ahlfors [1] and refined by Heins [6]. The main ingredient is a generalization of Schwarz' Lemma to metrics. The method was originally employed in the context of euclidean Bloch constants. We adapt the method so that it works in hyperbolic or spherical geometry. The essential features of the adaptation to spherical geometry occur in the work of Pommerenke [10]. His approach is analytic, while ours is geometric. In [8] we show that $B_{m, \Omega}(\alpha)>h_{m, \Omega}^{-1}(\alpha)$, where $h_{m, \Omega}^{-1}(\alpha)$ is an explicit function of $\alpha$. We give selected values of these functions below.

$$
\begin{gathered}
h_{m, \mathbf{B}}^{-1}(\alpha)=\left\{\begin{array}{cc}
\alpha\left[\frac{3}{8-5 \alpha^{2}+4\left(\alpha^{4}-5 \alpha^{2}+4\right)^{1 / 2}}\right]^{1 / 2}, & m=1, \\
\frac{\alpha}{1+\sqrt{1-\alpha^{2}}}, & m=\infty,
\end{array}\right. \\
h_{m, \mathrm{C}}^{-1}(\alpha)=\left\{\begin{array}{cc}
\frac{\alpha \sqrt{3}}{4}, & m=1, \\
\frac{\alpha}{2}, & m=\infty, \\
\alpha\left[\frac{3}{8+5 \alpha^{2}+4\left(\alpha^{4}+5 \alpha^{2}+4\right)^{1 / 2}}\right]^{1 / 2}, & m=1, \\
\frac{\alpha}{1+\sqrt{1+\alpha^{2}}}, & m=\infty .
\end{array}\right.
\end{gathered}
$$

Actually, in [8] these lower bounds are established in the context of meromorphic functions $f: X \rightarrow \Omega$, where $X$ is any hyperbolic Riemann surface and $f$ is suitably normalized at a fixed point of $X$. Also, the lower bounds for spherical Bloch constants improve bounds due to Tsuji [12], Greene and $\mathrm{Wu}[4]$ and Jorgenson [5].

We obtain upper bounds for the various Bloch constants by modifying the example of Ahlfors and Grunsky [2] to suit the geometry of the situation at hand. We refer the reader to [8] for details. We just note that for the hyperbolic and spherical Bloch constants examples are now needed for every admissible value of $\alpha$ rather than just for $\alpha=1$ as is the case for euclidean Bloch constants.

These results have applications to meromorphic functions defined on $\mathbf{C}$. Let

$$
B_{m, \Omega}=\inf \left\{r_{\Omega}(f): f \in F_{m}(\mathbf{C}, \Omega)\right\}
$$


Of course, here we are only considering $\Omega=\mathbf{C}$ or $\mathbf{P}$. It is known that $B_{1, \mathbf{C}}=\infty$; that is, the Riemann surface of a nonconstant entire function contains arbitrarily large unramified disks. In [9] we show that

$$
\frac{1}{\sqrt{3}} \leqslant B_{1, \mathbf{P}} \leqslant \frac{1}{\sqrt{2}}, \quad B_{\infty, \mathrm{P}}=1
$$

We also obtain results for Bloch constants for meromorphic functions on compact Riemann surfaces in [9].

Added In Proof. Recently, the author was informed that E. Peschl (Über unverzweigte konforme Abbildungen, Österreich. Akad. Wiss. Math.-Natur. K1. Sitzungsber. II 185 (1976), 55-78) had also considered euclidean, hyperbolic and spherical Bloch constants for locally schlicht functions in the unit disk and that he had obtained the nonstrict inequality $B_{\infty, \Omega}(\alpha) \geqslant h_{\infty, \Omega}^{-1}(\alpha)$ by a different method.

\section{REFERENCES}

1. L. V. Ahlfors, An extension of Schwarz's lemma, Trans. Amer. Math. Soc. 43 (1938), 359-364. $671-673$

2. L. V. Ahlfors and H. Grunsky, Über die Blochsche Konstante, Math. Z. 42 (1937),

3. A. Bloch, Les théorème de M. Valiron sur les fonctions entières et la théorie de l'uniformisation, Ann. Fac. Sci. Toulouse Math. (5) 3 (1925), 1-22.

4. R. E. Greene and $\mathrm{H}$. Wu, Bloch's theorem for meromorphic functions, Math. Z. 116 (1970), 247-257. 103.

5. V. Jorgensen, A remark on Bloch's theorem, Mat. Tidsskr. B 1952 (1952), 100-

6. M. Heins, On a class of conformal metrics, Nagoya Math. J. 21 (1962), 1-60.

7. E. Landau, Über die Blochsche Konstante und zwei verwandte Weltkonstanten,

Math. Z. 30 (1929), 608-634.

8. C. D. Minda, Bloch constants, J. Analyse Math. (to appear).

9. - Bloch constants for meromorphic functions, Math. Z. (to appear).

10. Ch. Pommerenke, Estimates for normal meromorphic functions, Ann. Acad. Sci. Fenn. Ser. A I Math. 476 (1970), 10 pp.

11. - On Bloch functions, J. London Math. Soc. (2) 2 (1970), 689-695. 170-171.

12. M. Tsuji, On an extension of Bloch's theorem, Proc. Imp. Acad. Japan 18 (1942),

DEPARTMENT OF MATHEMATICAL SCIENCES, UNIVERSITY OF CINCINNATI, CINCINNATI, OHIO 45221 